\title{
Spatial manipulation of nanoacoustic waves with nanoscale spot sizes
}

\author{
KUNG-HSUAN LIN ${ }^{1 \dagger}$, CHIH-MING LAI ${ }^{2}$, CHANG-CHI PAN ${ }^{3}$, JEN-INN CHYl ${ }^{3}$, JIN-WEI SHI ${ }^{3}$, \\ SHIH-ZE SUN ${ }^{1}$, CHIEH-FENG CHANG ${ }^{1}$ AND CHI-KUANG SUN ${ }^{1,4 *}$
}

\author{
1Department of Electrical Engineering and Graduate Institute of Photonics and Optoelectronics, National Taiwan University, Taipei 10617, Taiwan \\ ${ }^{2}$ Department of Electronic Engineering, Ming Chuan University, Taoyuan 33348, Taiwan \\ ${ }^{3}$ Department of Electrical Engineering, National Central University, Jhongli 32054, Taiwan \\ ${ }^{4}$ Research Center for Applied Sciences, Academia Sinica, Taipei 115, Taiwan \\ tPresent address: Department of Chemistry, Massachusetts Institute of Technology, Cambridge, Massachusetts 02139, USA \\ *e-mail: sun@cc.ee.ntu.edu.tw
}

Coherent acoustic phonons are generated at terahertz frequencies when semiconductor quantum-well nanostructures are illuminated by femtosecond laser pulses ${ }^{1-9}$. These phonons-also known as nanoacoustic waves-typically have wavelengths of tens of nanometres, which could prove useful in applications such as non-invasive ultrasonic imaging ${ }^{10-12}$ and sound amplification by the stimulated emission of radiation $^{13}$. However, optical diffraction effects mean that the nanoacoustic waves are produced with spot sizes on the micrometre scale. Near-field optical techniques can produce waves with smaller spot sizes, but they only work near surfaces ${ }^{14}$. Here, we show that a far-field optical techniquewhich suffers no such restrictions-can be used to spatially manipulate the phonon generation process so that nanoacoustic waves are emitted with lateral dimensions that are much smaller than the laser wavelength. We demonstrate that nanoacoustic waves with wavelengths and spot sizes of the order of $10 \mathrm{~nm}$ and $100 \mathrm{~nm}$, respectively, can be generated and detected.

The spatial manipulation of nanoacoustic waves (NAWs) is based on the saturation of the process that generates coherent phonons in piezoelectric semiconductor nanostructures ${ }^{15}$. As shown in Fig. 1a, when a femtosecond excitation laser pulse is used to generate photocarriers (electrons and holes) in the nanostructure, longitudinal NAWs are generated with a wavelength that is determined by the periodicity of the nanostructure ${ }^{8}$. The generation process can be modelled by a loaded-string model as ${ }^{16-19}$

$$
\frac{\partial^{2} u_{3}(\mathbf{r})}{\partial t^{2}}-V^{2} \frac{\partial^{2} u_{3}(\mathbf{r})}{\partial z^{2}}=D(\mathbf{r}, t)
$$

where $u_{3}(\mathbf{r})$ represents the longitudinal displacement field, $V$ is the sound velocity and $D(\mathbf{r}, t)$ is the driving function. In piezoelectric semiconductors, the driving functions are dominated by the piezoelectric effect, although other effects-including deformationpotential couplings ${ }^{16-20}$ _ are also involved.

When a laser pulse generates a low density of photocarriers in the quantum wells, the built-in piezoelectric field is screened due to the spatial separation of electrons and holes (Fig. 1b). This space-charge screening field causes the strain within the semiconductor to change as a function of time and this, in turn, leads to the formation of NAWs that travel away from each other in opposite directions ${ }^{18-20}$. For high carrier densities, however, the screening field produced by the photocarriers will be comparable to the built-in piezoelectric field, so it can no longer be treated as a perturbation to the built-in field ${ }^{16-19}$.

Here, we recalculate the coherent phonon generation efficiency for different two-dimensional (2D) photocarrier densities, which is the axial integral of the three-dimensional (3D) carrier densities (see Supplementary Information for details). As illustrated in Fig. 1c, when the screening field produced by the photocarriers is comparable to the built-in piezoelectric field, the electrons and holes move much closer to each other in the quantum wells (Fig. 1c). This means that the space-charge screening field that is the driving force behind the generation of the NAWs will not increase linearly with the $2 \mathrm{D}$ photocarrier density and will start to saturate (Fig. 1d,e). The threshold 2D carrier density for saturation in the InGaN/GaN quantum wells used in our experiments is $\sim 4 \times 10^{12} \mathrm{~cm}^{-2}$ (ref. 15).

We can exploit this saturation effect to manipulate the transverse spatial distribution of the phonon driving force by using a different laser pulse to pre-inject a non-uniform distribution of photocarriers. To generate an acoustic nanospot, for example, we can pre-inject carriers with a doughnut-shaped distribution (Fig. 2a; ref. 21). Owing to the saturation effect, the phonon generation efficiency is lower in the outer region, and higher at the centre, so the spot size of the NAWs is reduced. Finally, in the absence of an optical method to detect NAWs with spot sizes smaller than the optical diffraction limit, we manipulate the acoustic spot along the $x$ axis, and use the nanoultrasonic technique ${ }^{12}$ to estimate the spot size.

We used a binary phase plate to shape the pre-injection laser pulse with a spot size slightly larger than that of the excitation laser pulse $\mathrm{e}^{21}$. After carriers were pre-injected with a distribution of optical intensity as shown in Fig. 2b, the diffraction-limited optical excitation pulse (Fig. 2c) was focused into the InGaN multiple quantum well (MQW) region for exciting NAWs. With a proper carrier density to saturate the driving force in the periphery region out of $x=0$, it is expected that the excited 

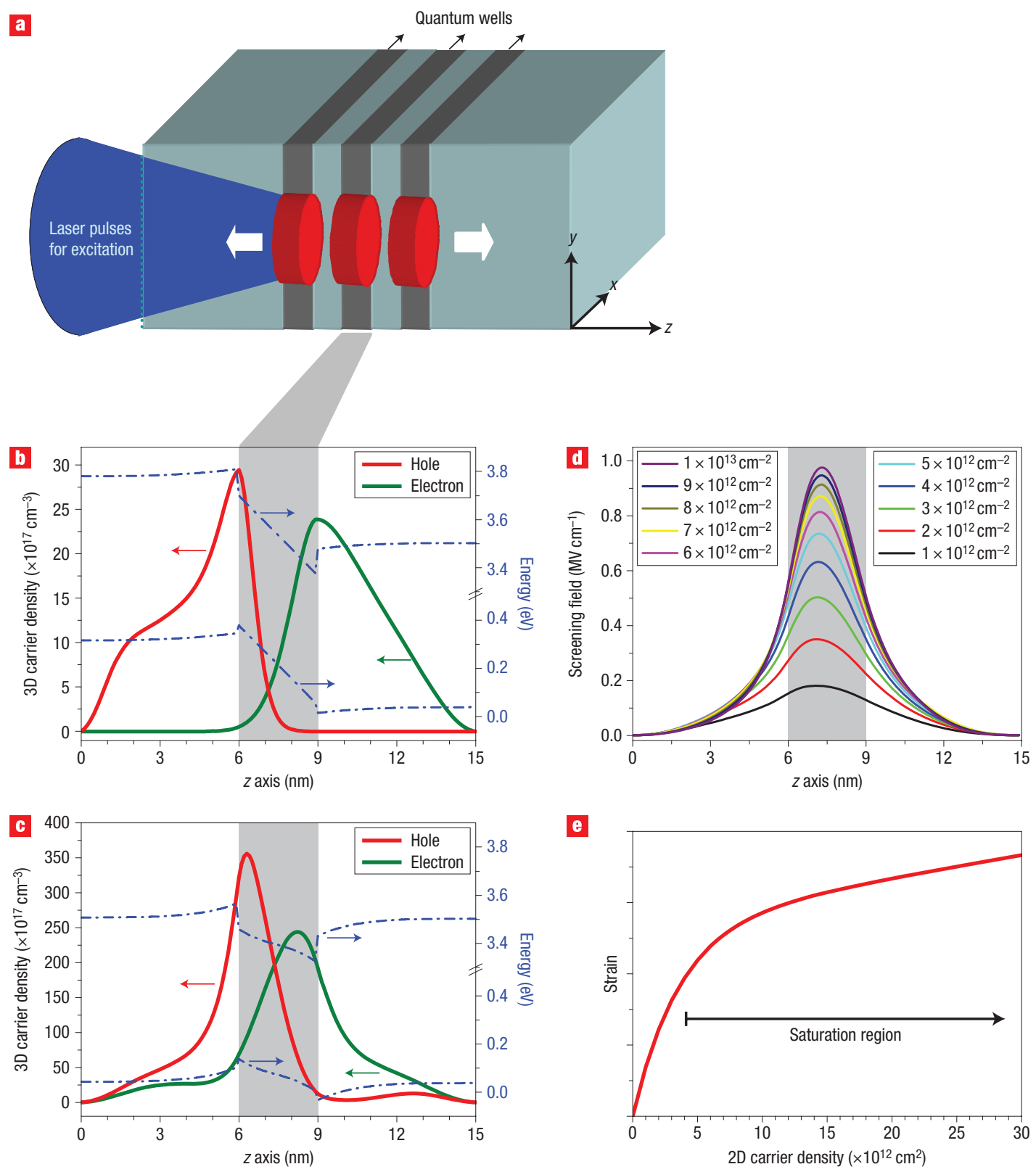

e

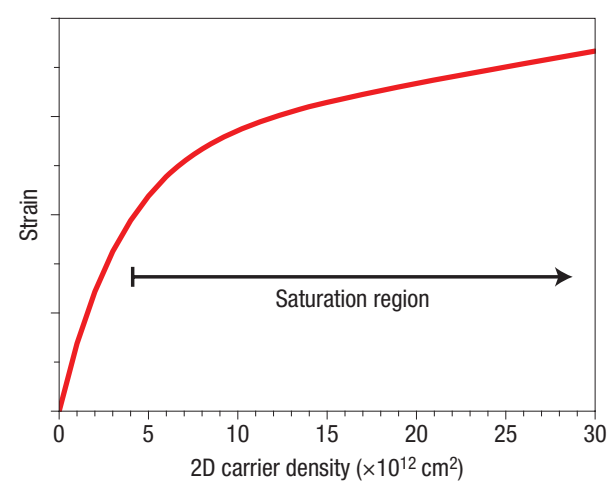

Figure 1 Coherent acoustic phonon generation in piezoelectric semiconductor nanostructures. a, When femtosecond optical pulses (blue cone) illuminate a quantum-well structure, it can generate coherent acoustic phonons (also known as nanoacoustic waves; white arrows) with wavelengths of $10 \mathrm{~nm}$ or less, as explained in the main text. The wavelength is determined by the period of the quantum wells ${ }^{8}$. In our experiment 7-nm-thick InGaN quantum wells (dark grey) are sandwiched between GaN layers (pale green). b. From our calculations, with a low $2 \mathrm{D}$ carrier density $\left(10^{12} \mathrm{~cm}^{-2}\right)$ in the quantum wells, the electron (green) and hole (red) wavefunctions are well separated due to the built-in piezoelectric field. The conduction band and valence band are shown in dot-dashed lines. c, For a higher carrier density $\left(10^{13} \mathrm{~cm}^{-2}\right)$, the calculated electron and hole wavefunctions move closer. $\mathbf{d}$, Calculated space-charge screening field distributions for 2D carrier densities between $1 \times 10^{12} \mathrm{~cm}^{-2}$ and $1 \times 10^{13} \mathrm{~cm}^{-2}$. e. For low carrier densities the strain increases linearly with the carrier density, but above about $4 \times 10^{12} \mathrm{~cm}^{-2}$ it starts to saturate. This curve is a fit to experimental data from ref. 15 .

acoustic spot size in the $x$ axis (Fig. 2d) should be smaller than that without pre-manipulation of the coherent phonon driving force (Fig. 2e). For the simulation result shown in Fig. 2d, we neglected the modulated optical absorption due to the existing carriers from pre-injection pulses. (See Supplementary Information for a detailed discussion of this secondary effect and description of the simulation.)

To measure the lateral acoustic spot size after manipulation, we etched a structure with a sharp edge in the cap layer on top of the nanoacoustic transducer (InGaN MQWs), as shown in Fig. 3a. After NAWs were generated, we used another femtosecond optical probe pulse to monitor the time delay for echoed NAWs back into the nanoacoustic transducer, the same as the onedimensional (1D) ultrasonic $\operatorname{scan}^{12}$ (called the A-scan ${ }^{11}$ ) measurement based on the 'pulse and echo' method ${ }^{8,10-12}$, to resolve the depth information (in the $z$ axis) of the tested sample. If the NAW spot is reflected from the edge of the striped pattern, the echoed waves will be split into two parts due to the sharp 

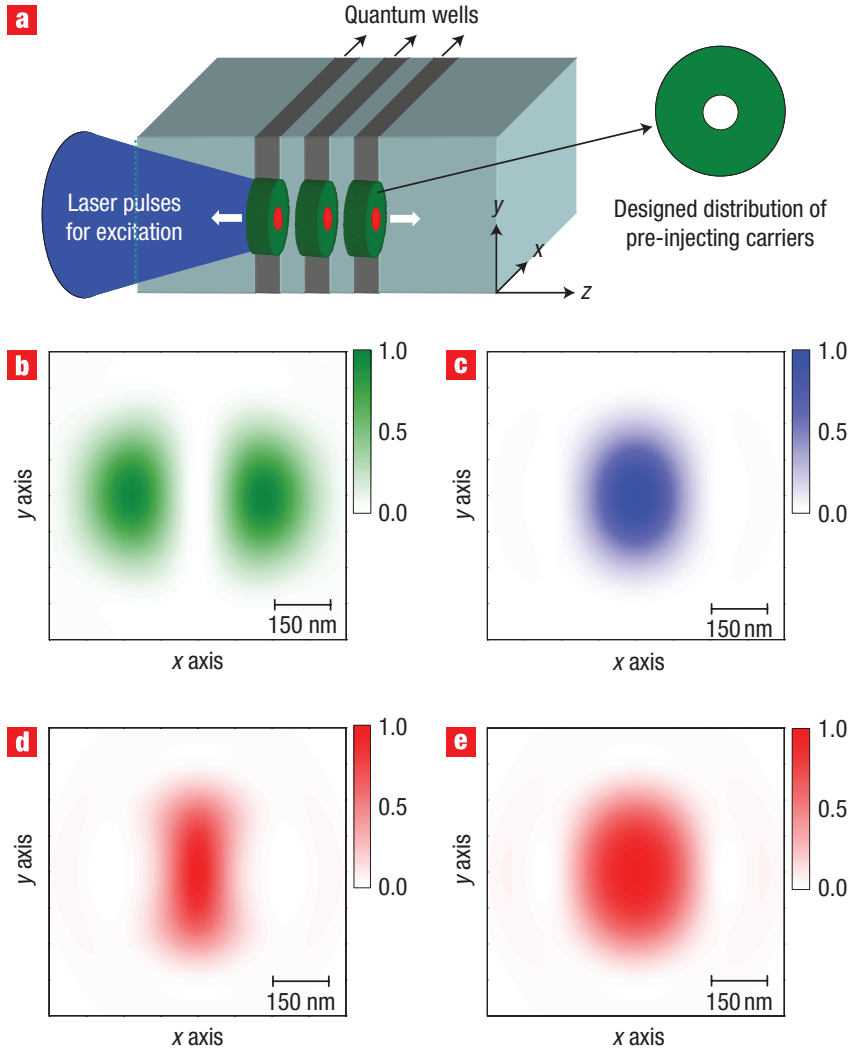

Figure 2 Spatial manipulation of coherent acoustic phonon generation.

a, Before the laser pulses excite the nanoacoustic waves (white arrows), one can pre-inject a designed photocarrier distribution (green) to reduce the lateral dimensions of the NAWs, as explained in the main text. b,c, Simulations predict that if the pre-injection pulses and the excitation pulses have the normalized profiles shown, it will be possible to reduce the width of the acoustic spot along the $x$ axis. $\mathbf{d}$, Acoustic intensity profile after spatial manipulation. In the simulations the peak of the carrier density is $7.5 \times 10^{12} \mathrm{~cm}^{-2}$ for both pulses, and the strain and 2D carrier density are related as shown in Fig. $1 \mathrm{e}$. The simulations also assume that the distribution of the 2D carrier density follows the distribution of the optical excitation intensity. e, Acoustic intensity profile without spatial manipulation. In the absence of the pre-injection pulse, the acoustic spot will be wider.

edge. A part of the echoed waves (denoted EW-1) returns back to the acoustic transducer earlier, whereas the other part (denoted EW-2) returns later. It should be noted that although these two echoes return at different time delays, they could both be measured in the acoustic transducer due to the diffractionlimited area of the optical detection pulses.

Without the pre-injecting pulse for driving force manipulation, the $2 \mathrm{D}$ nanoultrasonic $\mathrm{B}$ scans ${ }^{11}$ ( $x$ axis position-dependent $1 \mathrm{D}$ nanoultrasonic scans) were measured as shown in Fig. 3b. A detailed description of a typical trace can be found elsewhere ${ }^{8}$. At the scanning position of $x=-260 \mathrm{~nm}$, the $0.5 \mathrm{THz}$ NAWs were echoed back to the acoustic transducer with a $\sim 17 \mathrm{ps}$ time delay (EW-1). However, when the scanning position was $x=+240 \mathrm{~nm}$, the echoed time delay was $\sim 30 \mathrm{ps}$ (EW-2). Moving the scanning positions from $x=-260 \mathrm{~nm}$ to $+240 \mathrm{~nm}$, the echoed NAWs were split due to the sharp edge, although the EW-1 signal decreased and the EW-2 signal increased. We used a bandpass filter ${ }^{8}$ to process the traces and obtained the peak values of EW-1 and EW-2 signals. If 'EW2 percentage' is defined as the peak value of EW-2 divided by the sum of the amplitudes of the EW-2 and EW-1 signals (in each A-scan), a plot of EW2 percentage as a function of scanned $x$ position (as shown in Fig. 3d) reflects the acoustic spot size in the $x$ direction. With an optical excitation wavelength of $400 \mathrm{~nm}$ and an objective with 0.9 numerical aperture (NA), we successfully generated an acoustic spot with a lateral full-width at half-maximum (FWHM) spot size of $300 \mathrm{~nm}$ (after a gaussian fit as shown by the solid line in Fig. 3d) close to the transform-limited optical spot size.

We then used the manipulated NAW source to image the same structure. As shown in Fig. 3c, the echoes were dominated by EW-1 signals at $x=-130 \mathrm{~nm}$, whereas the echoes were dominated by EW-2 signals at $x=+120 \mathrm{~nm}$. When the driving force is manipulated, the variation of EW2 percentage as a function of position along the $x$ axis becomes much sharper, and the acoustic spot size is greatly reduced in this direction, as shown in Fig. 3e. With a gaussian fit shown as the solid line in Fig. 3e, the FWHM of the acoustic spot can be found to be reduced from $300 \mathrm{~nm}$ to $140 \mathrm{~nm}$ in the $x$ axis.

For a B-scan image (amplitude distribution of the echoed signals in the $z-x$ section), the lateral resolution is determined by the spot size of the applied acoustic pulses. Fig. $3 \mathrm{f}$ and g shows the original nanoultrasonic B-scan images of the GaN sharp edge, which were used to obtain the EW2 percentages for Fig. 3d and e, without and with the pre-injecting pulses for spatial manipulation, respectively. The two parallel white dotted lines label the $x$ positions where the EW2 percentages are 0.25 and 0.75 , respectively. Comparing these two nanoultrasonic B-scan images of the GaN sharp edge, it is apparent that because of the reduced acoustic spot size, the lateral range where the EW-1 and EW-2 echoes co-exist for a fixed $x$ position is reduced in Fig. 3g. The lateral $(x)$ resolution is thus higher when using the manipulated nanoacoustic source. For both Fig. $3 \mathrm{f}$ and $\mathrm{g}$ images, the axial $(z)$ resolutions are both on the order of $20 \mathrm{~nm}$, which is related to the acoustic pulsewidth as revealed in a previous study ${ }^{12}$. To further reduce the acoustic spot size, a stronger saturation effect is desired. In our current system, as revealed in Fig. 1e, phonon generation will not be completely saturated with even higher carrier density as a result of the deformation coupling force and non-perfect screening of the built-in electric field in the quantum wells. Moreover, spatial manipulation of coherent phonon generation should not be limited along one axis. Phonon generation can be engineered by manipulating the phase and polarization of the optical pulses, for example as a doughnut shape ${ }^{21}$, to pre-inject a patterned carrier distribution in the nanoacoustic transducer.

In summary, we have generated NAWs with spot sizes that are smaller than the optical diffraction limit of the laser excitation pulse used to generate the wave. Our technique also makes it possible to control the temporal profile of the waves ${ }^{15,22,23}$. This ability to manipulate terahertz acoustic phonon generation in the both temporal and spatial domains could prove useful in many areas of 3D nanoultrasonics.

\section{METHODS}

\section{SAMPLE PREPARATION}

The sample has a three-period $70 \AA / 70 \AA \operatorname{In}_{0.23} \mathrm{Ga}_{0.77} \mathrm{~N} / \mathrm{GaN}$ MQW structure grown on 3.4- $\mu \mathrm{m}$-thick $\mathrm{GaN}$ and $500-\mu \mathrm{m}$-thick sapphire substrates. On top of the MQW, the $100-\mathrm{nm}$-thick GaN cap layer has a periodic stripe structure acting as the imaging target under test. The stripe pattern has a period of $3 \mu \mathrm{m}$ including a $1-\mu \mathrm{m}$-wide etched region, and the etched depth is $\sim 55 \mathrm{~nm}$. The 'edge' of the stripes rises within $20 \mathrm{~nm}$, estimated by a scanning electron microscope (SEM).

The optical pattern as shown in Fig. 2b was achieved by a phase plate with phase shift $\pi$ between the $x<0$ region and the $x>0$ region. An oxygenic silicon nitride $(n=1.53)$ layer with a thickness of $377 \mathrm{~nm}$ was grown on a sapphire 

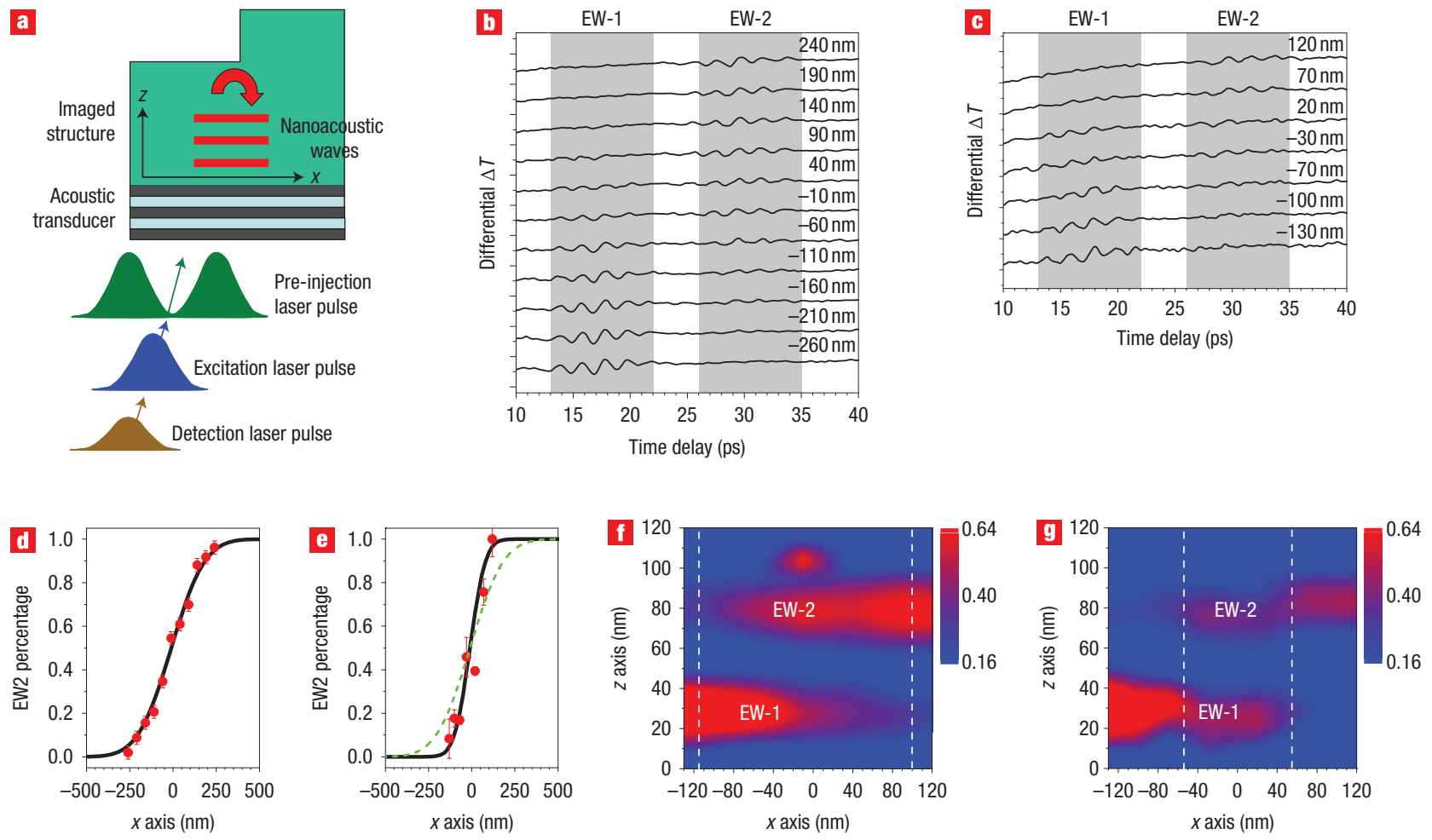

Figure 3 Experimental demonstration of spatially manipulated coherent phonon generation. a, The NAWs generated in the quantum-well structure were used to image an object with a sharp edge. The sharpness of the edge in the image indicates the spot size of the acoustic wave source. The imaging process involves three laser pulses: the pre-injection pulse (green), the excitation pulse (blue) and the detection pulse (brown). b,c, One-dimensional ultrasonic traces at different $x$ axis positions show the variation of the echoed wave amplitudes from both surfaces (EW-1 and EW-2) for NAWs from a source without pre-injection (b) and a source with pre-injection (c). d,e, The edge in the plot of EW2 percentage (red solid circles) versus position for a source without pre-injection (d), is not as sharp as that for a source with pre-injection (e). (The solid lines are gaussian fits, and the green dashed line in $\mathbf{e}$ shows the curve from $\mathbf{d}$ for comparison.) $\mathbf{f}, \mathbf{g}$, The nanoultrasonic B-scan images of the GaN sharp edge without pre-injection (f) and with pre-injection $(\mathbf{g})$ clearly reveal that the lateral $(x)$ resolution is improved by spatial manipulation of the piezoelectric driving force. These two images are presented with linear-scaled false colours. The two parallel white dotted lines label the $x$ positions where the EW2 percentages are 0.25 and 0.75 , respectively.

substrate to introduce a delayed $\pi$-phase shift for the optical wavelength of $400 \mathrm{~nm}$. For shaping the optical spot pattern, the dividing line was parallel to the light polarization.

\section{EXPERIMENTAL SETUP FOR MANIPULATING COHERENT ACOUSTIC PHONON GENERATION} The optical pulses were generated from a Ti:sapphire oscillator with a repetition rate of $\sim 76 \mathrm{MHz}$ (Mira, Coherent Incorporation). The output laser pulses were frequency doubled by a beta barium borate crystal and were split into three beams for manipulation (pre-injecting), excitation (pump) and detection (probe). The frequency-doubled $(400 \mathrm{~nm}$ ) pulses had a pulsewidth of $\sim 250 \mathrm{fs}$ and the FWHM of the output spectral bandwidth was $2.5 \mathrm{~nm}$. The power of the pump beam and manipulating beam were $4 \mathrm{~mW}$ and $8 \mathrm{~mW}$, respectively. Three beams were combined by a polarization beamsplitter cube and were collinearly focused into the sample with an NA $=0.9$ objective lens. The advanced time of the pre-injecting pulse to the pump pulse was $5 \mathrm{ps}$, and the leaving time of the NAWs from the MQW was $\sim 4$ ps. The samples were placed on a $2 \mathrm{D}$ nanopositioner (P-733.2CL, PI) on the stage of an optical microscope (IX70, Olympus) for lateral $x y$-scan. The piezo-nanopositioner provided a spatial resolution $<0.3 \mathrm{~nm}$ under the electro-closed-loop control.

To increase the signal-to-noise ratio, the transmission changes of the optical probe pulse as a function of pump-probe time delay were recorded by using a 'double chopping' technique. We modulated the optical pump beam using an acousto-optic modulator with a frequency of $500 \mathrm{kHz}$. The optical probe beam was also modulated by a mechanical chopper wheel with a frequency of $880 \mathrm{~Hz}$. The electrical signal from the photomultiplier tube (illuminated by the transmitted pump and probe pulses) was demodulated by a radio-frequency lock-in amplifier (for $500 \mathrm{kHz}$ ) and a regular lock-in amplifier (for $880 \mathrm{~Hz}$ ). Note that in this experimental setup, the pre-injecting beam was not chopped.
The demodulated transmission variations of the probe pulse as a function of time delay were only sensitive to the NAWs initiated by the pump beam.

Received 25 June 2007; accepted 24 August 2007; published 14 October 2007.

\section{References}

1. Yamamoto, A., Mishina, T., Masumoto, Y. \& Nakayama, M. Coherent oscillation of zone-folded phonon modes in GaAs/AlAs superlattices. Phys. Rev. Lett. 73, 740-743 (1994).

2. Bartels, A., Dekorsy, T., Kurz, K. \& Kohler, K. Coherent zone-folded longitudinal acoustic phonons in semiconductor superlattices: excitation and detection. Phys. Rev. Lett. 82, 1044-1047 (1999).

3. Thomsen, C., Grahn, H. T., Maris, H. J. \& Tauc, J. Surface generation and detection of phonons by picosecond light-pulses. Phys, Rev. B 34, 4129-4138 (1986).

4. Sun, C.-K., Liang, J.-C. \& Yu, X.-Y. Coherent acoustic phonon oscillations in semiconductor multiple quantum wells with piezoelectric fields. Phys. Rev. Lett. 84, 179-182 (2000).

5. Trigo, M., Bruchhausen, A., Fainstein, A., Jusserand, B. \& Thierry-Mieg, V. Confinement of acoustical vibrations in a semiconductor planar phonon cavity. Phys. Rev. Lett. 89, 227402 (2002).

6. Matsuda, O., Wright, O. B., Hurley, D. H., Gusev, V. E. \& Shimizu, K. Coherent shear phonon generation and detection with ultrashort optical pulses. Phys. Rev. Lett. 93, 095501 (2004).

7. Bargheer, M. et al. Coherent atomic motions in a nanostructure studied by femtosecond X-ray diffraction. Science 306, 1771 (2004).

8. Lin, K.-H. et al. Optical piezoelectric transducer for nano-ultrasonics. IEEE Trans. Ultrason Ferroelectr. Freq. Control 52, 1404-1414 (2005).

9. Lanzillotti-Kimura, N. D., Fainstein, A., Lemaitre, A. \& Jusserand, B. Nanowave devices for terahertz acoustic phonons. Appl. Phys. Lett. 88, 083113 (2006).

10. Tas, G., Loomis, J. J., Maris, H. J., Bailes, A. A. \& Seiberling L. E. Picosecond ultrasonics study of the modification of interfacial bonding by ion implantation. Appl. Phys. Lett. 72, 2235 (1998).

11. Andriamonje, G. et al. Scanning laser ultrasonics experiments for in situ nondestructive analysis of integrated circuits. IEEE Trans. Device Mater. Reliab. 5, 564 (2005).

12. Lin, K.-H. et al. Two-dimensional nanoultrasonic imaging by using acoustic nanowaves. Appl. Phys. Lett. 89, 043106 (2006)

13. Kent, A. J. et al. Acoustic phonon emission from a weakly coupled superlattice under vertical electron transport: observation of phonon resonance. Phys. Rev. Lett. 96, 215504 (2006).

14. Vertikov, A., Kuball, M., Nurmikko, A. V. \& Maris, H. J. Time-resolved pump-probe experiments with subwavelength lateral resolution. Appl. Phys. Lett. 69, 2465-2467 (1996). 
15. Yu, C.-T. et al. Generation of frequency-tunable nanoacoustic waves by optical coherent control. Appl. Phys. Lett. 87, 093114 (2005).

16. Sanders, G. D., Stanton, C. J. \& Kim, C. S. Theory of coherent acoustic phonons in $\operatorname{In}_{x} \mathrm{Ga}_{1-x} \mathrm{~N} / \mathrm{GaN}$ multiple quantum wells. Phys. Rev. B 64, 235316 (2001)

17. Sanders, G. D., Stanton, C. J. \& Kim, C. S. Erratum: Theory of coherent acoustic phonons in $\mathrm{In}_{x} \mathrm{Ga}_{1-x} \mathrm{~N} / \mathrm{GaN}$ multiple quantum wells. Phys. Rev. B 66, p079903(E) (2002).

18. Chern, G.-W., Lin, K.-H. \& Sun, C.-K. Transmission of light through quantum heterostructures modulated by coherent acoustic phonons. J. Appl. Phys. 95, 1114-1121 (2004).

19. Chern, G.-W., Sun, C.-K., Sanders, G. D. \& Stanton, C. J. Generation of coherent acoustic phonons in nitride-based semiconductor nanostructures. Top. Appl. Phys. 92, 339-394 (2004).

20. Lin, K.-H., Yu, C.-T., Wen, Y.-C. \& Sun, C.-K. Generation of picosecond acoustic pulses using a p-n junction with piezoelectric effects. Appl. Phys. Lett. 86, 093110 (2005).

21. Klar, T. A., Engel, E. \& Hell, S. W. Breaking Abbé's diffraction resolution limit in fluorescence microscopy with stimulated emission depletion beams of various shapes. Phys. Rev. E 64, 066613 (2001).

22. Sun, C.-K. et al. Coherent optical control of acoustic phonon oscillations in InGaN/GaN multiplequantum-wells. Appl. Phys. Lett. 78, 1201-1203 (2001).
23. Chern, G.-W., Lin, K.-H., Huang, Y.-K. \& Sun, C.-K. Spectral analysis of high-harmonic coherent acoustic phonons in piezoelectric semiconductor multiple quantum wells. Phys. Rev. B 67, 121303 (R) (2003).

Acknowledgements

The authors would like to thank Yuen-Lin Tsai and Wen-Pin Huang for processing the sample. This work was sponsored by the National Science Council of Taiwan under Grant No. 95-2120-M-002-013. Correspondence and requests for materials should be addressed to C.-K.S.

Supplementary information accompanies this paper on www.nature.com/naturenanotechnology.

Author contributions

C.-K.S. conceived and conducted the studies. K.-H.L. designed the experiments. K.-H.L. and C.-M.L. performed the simulation. K.-H.L., S.-Z.S. and C.-F.C. performed the experiments. K.-H.L. and C.-K.S. analysed the data. C.-C.P., J.-I.C. and J.-W.S. contributed the samples. K.-H.L. and C.-K.S. wrote the paper.

Reprints and permission information is available online at http://npg.nature.com/reprintsandpermissions/ 